\title{
A importância da TCFC no diagnóstico e localização de dentes supranumerários
}

\author{
The importance of CBCT in supernumerary teeth diagnosis and location
}

Nathália de Alencar Valente

Ortodontista pelo HCA-FAB

Aluna de Especialização em Radiologia Odontológica da ABO-RJ

\section{Bárbara Monteiro Soares}

Estomatologista pela Unigranrio

Eduardo José da Costa Santos

Professor de Radiologia Odontológica da ABO-RJ

Milena Bortolotto Felippe Silva

Coordenadora de Radiologia S.L. Mandic

Resumo

Dentes supranumerários são achados frequentes, podendo encontrar-se inclusos nas arcadas. As radiografias convencionais são utilizadas para 0 diagnóstico e permitem a visualização da posição dentária e a relação com estruturas vizinhas. Entretanto, essas modalidades de imagem apresentam limitações que podem comprometer o diagnóstico e o tratamento. $\mathrm{O}$ uso da imagem tridimensional obtida pela Tomografia Computadorizada de Feixe Cônico (TCFC) supera muitas dessas desvantagens, tornando essa técnica superior às convencionais, em casos envolvendo dentes inclusos e/ou impactados. 0 objetivo deste trabalho é apresentar uma revisão de literatura destacando às vantagens da TCFC em relação às imagens bidimensionais no diagnóstico de hiperdontia.

Palavras-chave: tomografia computadorizada de feixe cônico; dentes supranumerários; radiografia.

\section{ABstract}

Supernumerary teeth are common findings, can be found included in the arcades. Conventional radiographs are used for the diagnosis and allow visualization of dental position and the relationship with surrounding structures. However, these imaging modalities have limitations which could compromise the diagnosis and treatment. The use of three-dimensional image obtained by Cone Beam Computed Tomography (CBCT) overcomes many of these disadvantages, making this technique superior than conventional, in cases involving teeth included and / or impacted. The objective of this study is to present a literature review with emphasis on the advantages of CBCT compared to two-dimensional images in diagnosis of hyperdontia.

Keywords: cone beam computed tomography; supernumerary teeth; radiograph.

\section{Introdução}

O $s$ exames de imagem são componentes essenciais no plano de tratamento em Odontologia, especialmente nos casos que envolvem estruturas em localização intraóssea nas arcadas dentárias, como ocorre frequentemente na hiperdontia. Dentes supranumerários podem apresentar-se em íntima relação com as demais estruturas anatômicas adjacentes, como outros elementos dentários, seio maxilar, fossa nasal e canal mandibular. Além disso, variações anatômicas podem estar presentes e o diagnóstico por imagem se faz essencial na visualização de tais alterações, no intuito de atingir melhores resultados e gerando a menor morbidade possível ao paciente, durante um possível procedimento cirúrgico. Dentre os exames de imagem mais utilizados pelo dentista, a radiografia panorâmica tem papel de destaque, pois possibilita uma ampla visualização das estruturas com baixa dose de radiação. Entretanto, essa técnica possui algumas desvantagens como: apresentar sobreposição de estruturas, ampliação e distorção ao longo da imagem, entre outras. O surgimento da Tomografia Computadorizada de Feixe Cônico (TCFC) expandiu o campo da radiologia oral e maxilofacial, pois supera as desvantagens das radiografias convencionais, obtendo informações tridimensionais das estruturas, sem sobreposições e com alta precisão (1). Medidas exatas, localização precisa, abordagem cirúrgica ideal e preservação de estruturas são possíveis com a utilização do exame tridimensional. Assim, nos casos de múltiplos elementos supranumerários, a TCFC se destaca como uma importante ferramenta para a definição de um diagnóstico preciso e na correta escolha da técnica cirúrgica a ser empregada.

\section{Revisão de Literatura}

Alterações de desenvolvimento do órgão dentário resultam em anormalidades estruturais do esmalte e/ou dentina, como anomalias de tamanho, número e forma. Esses defeitos podem ser resultado de fatores locais ou sistêmicos (2). A hiperdontia, uma alteração de número, resulta na formação de elementos supranumerários, que são dentes em uma quantidade maior do que o número considerado normal nas arcadas dentárias. GALLAS et al. (3) relatam a influência do fator genético no desenvolvimento de dentes supranumerários, visto que em alguns estudos são relatados casos de supranumerários em integrantes da mesma família por gerações diferentes. Os dentes supranumerários podem ser classificados em: rudimentares quando apresentam forma diferente do dente normal ou suplementares, quando apresentam morfologia normal. A frequência da hiperdontia é de $0,3 \%$ a 3,8\%, sendo que cerca de $90 \%$ a $98 \%$ ocorrem na maxila, mais comumente na região anterior (4).

ALMEIDA et al. (5) destacam as consequências que a presença de elementos supranumerários podem causar: devido à falta de espaço nas arcadas, muito frequentemente esses elementos apresentam-se retidos. As complicações que envolvem a presença desse defeito são apinhamento dental, impacção de dentes permanentes, má oclusão, erupção retardada e/ou ectópica, distúrbios na articulação temporomandibular, rotação dentária, formação de diastemas, deslocamentos dentários, reabsorções dentárias, comprometi- 
mento de enxertos ósseos alveolares, desarmonia funcional, problemas estéticos e desenvolvimento de patologias orais, como o cisto dentígero (5).

Para a prevenção de tais complicações, a remoção dos elementos é muitas vezes indicada. O diagnóstico para a decisão a ser tomada, bem como o planejamento da abordagem cirúrgica, são essenciais. PAROLIA et al. (6) ressaltam que a localização precisa dos elementos retidos, com detalhes anatômicos do seu entorno, e a visualização da anatomia da coroa e raízes permitem um adequado planejamento, diminuição do tempo cirúrgico, redução do estresse para o profissional e paciente. Para isso, é necessário que seja realizado um diagnóstico correto através de exames de imagens. A radiografia panorâmica é amplamente utilizada para decisões de tratamento, sendo muitas vezes o único exame de imagem solicitado ao paciente. Porém, apresenta as desvantagens de ser uma representação bidimensional de estruturas tridimensionais, causando sobreposições, além de apresentar pouco detalhe, ampliação desigual e distorção geométrica ao longo da imagem (7). Em casos de elementos supranumerários, principalmente inclusos, detalhes podem ser obscurecidos. A figura 1 apresenta um exemplo de radiografia panorâmica apresentando múltiplos elementos supranumerários.

GUPTA et al. (8) compararam imagens de radiografias panorâmicas de pacientes portadores da displasia cleidocraniana, síndrome que apresenta múltiplos elementos supranumerários, com imagens de TCFC. Destacou o fato de os elementos frequentemente estarem fora do plano de corte na técnica panorâmica, o que gerou grande dificuldade na localização destes no exame bidimensional. Além disso, a extração de múltiplos elementos resulta em áreas edêntulas e o exame tridimensional serve como guia para o planejamento reabilitador.

KATHERIA et al. (9) compararam imagens de radiografias panorâmicas de casos de múltiplos elementos supranumerários com imagens de TCFC. Concluíram que foi possível identificar a presença dos dentes inclusos através das duas modalidades diagnósticas, porém, o exame tridimensional forneceu maiores informações a respeito de sua localização, presença de reabsorções dentárias e detalhes anatômicos.

A Tomografia Computadorizada (TC tradicional) foi criada na década de 70 e adquire as imagens do corpo através de feixes de raios $\mathrm{X}$ reproduzindo imagens de uma secção do corpo nos três planos do espaço. Possui as vantagens principais de evidenciar as estruturas em profundidade e sem sobreposições, adquirindo as imagens através de "fatias" do corpo humano por meio de um feixe em forma de leque (10). Nos anos 90, foram desenvolvidos trabalhos utilizando a TCFC para uso em Odontologia. Essa tecno- logia representa o desenvolvimento de um tomógrafo relativamente pequeno e de menor custo, especialmente indicado para a região dentomaxilofacial. Esta técnica proveu à Odontologia a reprodução da imagem tridimensional dos tecidos mineralizados maxilofaciais, com mínima distorção e dose de radiação significantemente reduzida em comparação à TC tradicional (11). Seus aparelhos são menores e apresentam praticidade na confecção do exame e uma ótima definição de imagem. As imagens são adquiridas em segundos conforme a fonte e o receptor se movimentam ao redor do paciente, diminuindo a emissão de raios em direções indesejadas e sobreposições, em um feixe de formato cônico. Nessas aquisições as imagens são gravadas em linguagem DICOM (Digital Imaging and Communication in Medicine) o que permite a reconstrução por algoritmos específicos e nos diferentes planos da área escaneada, ou seja, a visualização de imagens axiais, coronais, sagitais e oblíquas, assim como em 3D (12). As figuras 2, 3 e 4 mostram alguns exemplos de cortes obtidos pela TCFC.

MOSSAZ et al. (13) avaliaram o desempenho da TCFC na localização e visualização de características morfológicas de elementos supranumerários, a frequência e extensão de reabsorção radicular em dentes adjacentes. Os autores concluíram que o exame foi eficaz em determinar ou grau de reabsorção de elementos associados a supranumerários impactados.

As características anatômicas dos elementos inclusos, como forma das raízes, da câmara pulpar e da coroa, são de extrema importância da definição do plano de tratamento. Segundo KAPILA et al. (14), a TCFC auxilia o profissional a decidir qual elemento deve ser removido baseado em sua anatomia.

Algumas alterações como fusões e reabsorções dentárias podem estar presentes na hiperdontia. ERICSON et al. (15) documentaram que a presença e a extensão de reabsorção radicular em dentes adjacentes a elementos impactados é difícil de ser avaliada através de radiografias convencionais e que a TCFC proveu melhor visualização facilitando avaliar a presença de reabsorção, morfologia radicular e dilacerações.

FERREIRA-JUNIOR et al. (16) relataram um caso de fusão entre um terceiro molar impactado e um paramolar em que a exodontia dos elementos estava indicada. Foi observado que a radiografia panorâmica não foi eficaz em revelar detalhes que só puderam ser observados com a TCFC. O exame tridimensional revelou uma fusão entre os elementos e íntima relação entre suas raízes e o canal mandibular.

Outra situação comum é a de dentes supranumerários que podem ser aproveitados e o tratamento endodôntico pode ser necessário. KATO et al. (17) relataram um caso de segundo molar fusionado com um paramolar em que o tratamento endodôntico estava indicado. Radiografias panorâmicas e TCFC 
foram obtidas e os autores concluíram que apenas com a tomografia computadorizada foi possível observar que havia conexão pulpar entre os elementos, o que foi imprescindível para o sucesso do tratamento endodôntico.

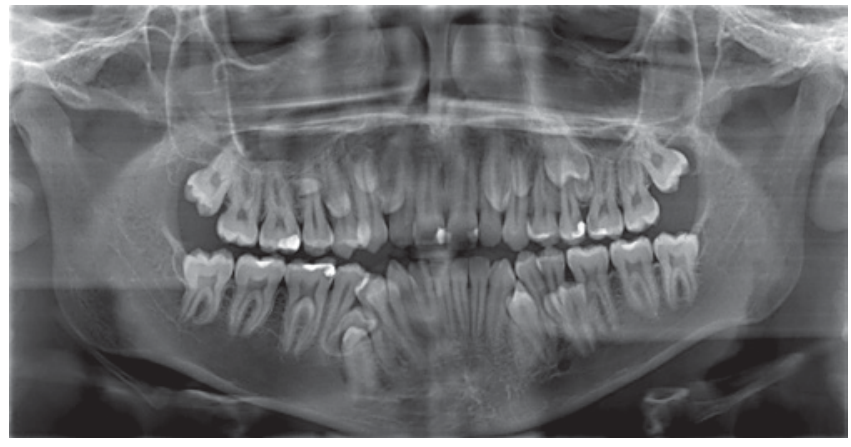

Figura 1. Exemplo de radiografia panorâmica apresentando múltiplos elementos supranumerários

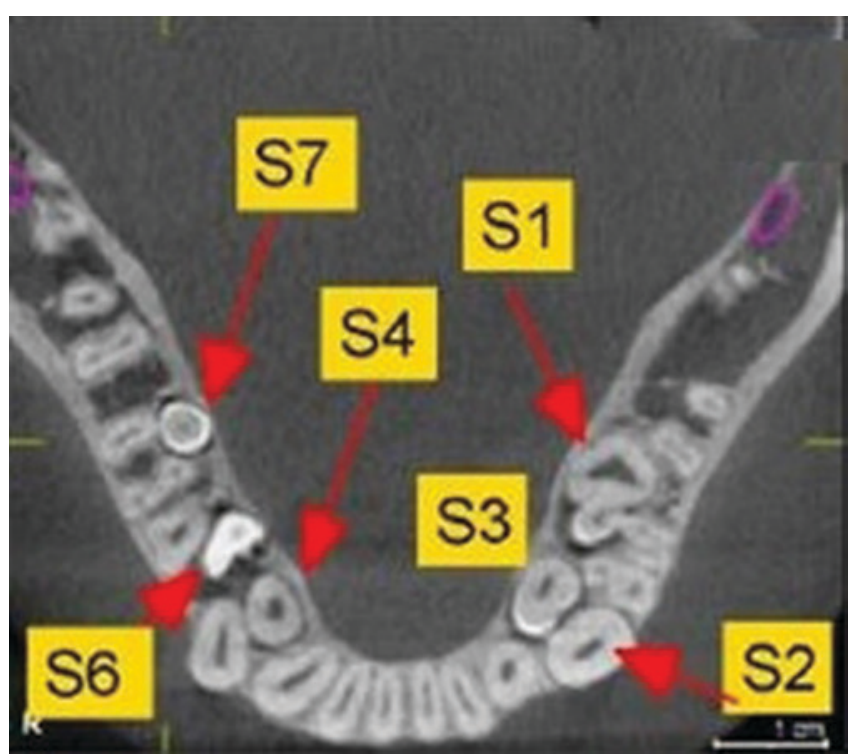

Figura 3. Corte axial de mandíbula apresentando a localização vestíbulo-lingual de múltiplos elementos supranumerários indicados pelas setas vermelhas

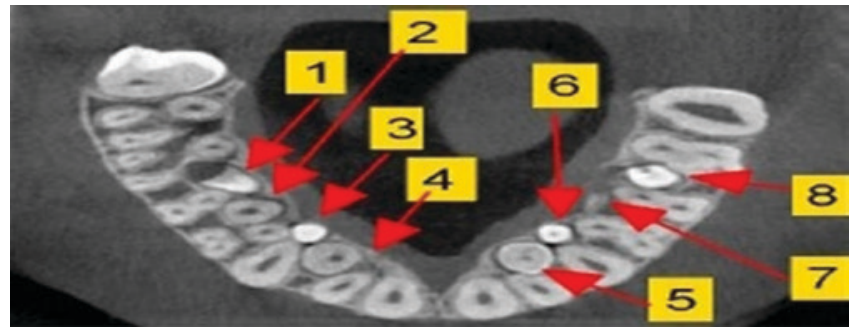

Figura 2. Imagem de TCFC. Corte axial de maxila apresentando a localização vestíbulo-lingual de múltiplos elementos supranumerários indicados pelas setas vermelhas

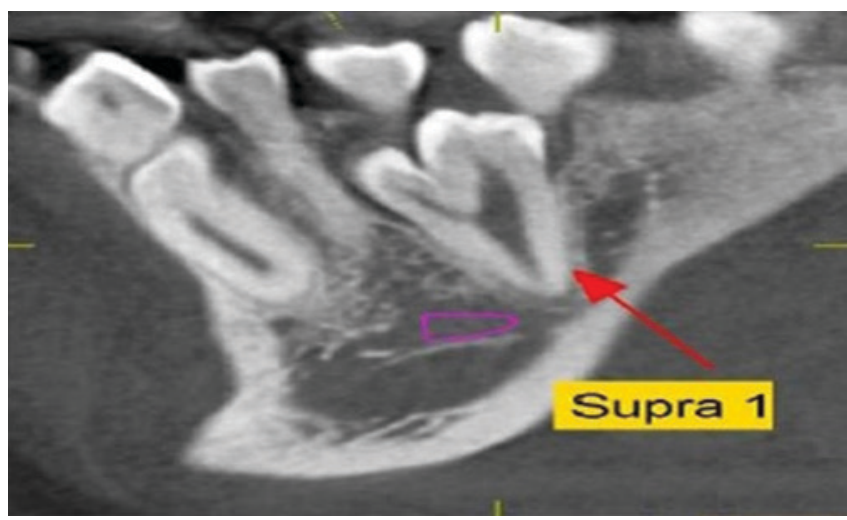

Figura 4. Corte oblíquo de mandíbula evidenciando alteração de um elemento supranumerário (indicado pela seta) que apresenta geminação. Tal alteração não é observada na radiografia panorâmica

\section{Discussão}

O exame radiográfico bidimensional faz parte da rotina do cirurgião-dentista sendo muitas vezes a base para decisões de tratamento. Radiografias panorâmicas são exames simples, de baixo custo, baixa dose, rápida aquisição e podem ser utilizadas em pacientes com dificuldade de abertura de boca (7). Porém, em algumas situações, suas limitações podem comprometer o sucesso do tratamento, o que pode ocorrer em casos de elementos supranumerários, principalmente quando estes estão inclusos nas bases ósseas.

A Tomografia Computadorizada de Feixe Cônico apresenta aplicações nas variadas especialidades odontológicas e tende a ser cada vez mais utilizada. Essa modalidade diagnóstica abrange apenas a região maxilofacial e permite a visualização de todas as estruturas anatômicas importantes desta área, bem como sua relação com os dentes. Assim, apresenta indicação no diagnóstico de hiperdontia. Esses dentes e sua relação com estruturas adjacentes podem ser visualizados através dos diferentes cortes tomográficos: axial, coronal, sagital, transaxial e oblíquo, ou seja, em três dimensões, superando a limitação do exame bidimensional (18).

REICHARDT et al. (19), entretanto, ressaltam que uma desvantagem desse exame é a baixa resolução para visualização de tecidos moles. Entretanto, deve ser considerado que a maioria dos casos de interesse na região maxilofacial envolve tecidos duros. Outra desvantagem da TCFC é a presença de artefatos, que são mais comuns quando comparada com as radiografias convencionais. Artefatos de movimento do paciente podem produzir imagens duplicadas. Objetos metálicos, tais como restau- 
rações de amálgama, aparelhos ortodônticos e implantes também podem comprometer a correta visualização de estruturas através da produção de artefatos (20).

RODRIGUES et al. (21) consideram que o custo-benefício da exposição do paciente às radiações ionizantes deve ser levado em conta, estabelecendo protocolos adequados para cada tipo de avaliação e necessidade.

A dose de radiação efetiva da tomografia computadorizada odontológica varia de acordo com a marca comercial do aparelho e com as especificações técnicas selecionadas durante a tomada (campo de visão, tempo de exposição, miliamperagem e quilovoltagem) (22). Porém, de um modo geral, ela mostra-se significantemente reduzida em comparação à tomografia computadorizada tradicional (23). Quando comparada às radiografias convencionais, a dose de radiação da TCFC equivale a aproximadamente 4 a 15 vezes a dose de uma radiografia panorâmica (11).

GARIB et al. destacam que uma outra vantagem de um exame de TCFC é que profissional pode obter reconstruções de todas as tomadas radiográficas convencionais odontológicas (24).

Segundo FARMAN (25), o princípio de ALARA (As Low As Reasonably Achievable) é a base antes da prescrição de qualquer exame radiológico, de modo que o risco provocado pela exposição à radiação ionizante deve ser sempre confrontado pelo benefício em potencial desse exame para o paciente.

Assim, como pode ser observada na revisão apresentada, apesar da maior dose da TCFC comparada à radiografia convencional, a solicitação do exame tridimensional é justificada pela superioridade em fornecer informações imprescindíveis em casos de elementos supranumerários, prevenindo o desenvolvimento de problemas futuros.

\section{Conclusão}

A revisão de literatura apresentada mostra que em diversos casos de elementos supranumerários, informações cruciais para o sucesso do tratamento podem não ser visualizadas no exame bidimensional. Apesar do maior custo e maior dose de radiação a que o paciente é exposto com a utilização do exame tridimensional, este é justificado devido ao benefício que proporciona. A definição de possíveis variações anatômicas, topologia coronária, radicular e da câmara pulpar, auxílio na escolha dos elementos que devem ser aproveitados, presença de reabsorções e patologias, melhor abordagem cirúrgica, de tratamento endodôntico e prevenção de danos às estruturas adjacentes, destacam que este exame é indispensável no planejamento da hiperdontia. 


\section{Referências ::}

1. Affairs ADACoS. The use of cone-beam computed tomography in dentistry: an advisory statement from the American Dental Association Council on Scientific Affairs. The Journal of the American Dental Association. 2012;143(8):899-902.

2. Kapdan A, Kustarci A, Buldur B, et al. Dental anomalies in the primary dentition of Turkish children. European journal of dentistry. 2012;6(2):178-83

3. Gallas MM, Garcia A. Retention of permanent incisors by mesiodens: a family affair. British dental journal. 2000;188(2):63-4.

4. Sharma A, Singh VP. Supernumerary teeth in Indian children: a survey of 300 cases. International journal of dentistry. 2012;2012:745265.

5. Almeida T, Saavedra Junior J, Kawakami et al. Hiperdontia: relato de caso com 8 elementos supranumerários. Rev odontol Univ Cid São Paulo. 2010;22(1).

6. Parolia A, Kundabala M, Dahal M, et al. Management of supernumerary teeth. Journal of conservative dentistry: JCD. 2011;14(3):221-4.

7. White SC, Pharoah MJ. Oral radiology: principles and interpretation: Elsevier Health Sciences; 2014.

8. Gupta NS, Gogri AA, Kajale MM, et al. Cone-beam computed tomography: An inevitable investigation in cleidocranial dysplasia. Contemporary clinical dentistry. 2015;6(2):257-61.

9. Katheria BC, Kau CH, Tate R, et al. Effectiveness of impacted and supernumerary tooth diagnosis from traditional radiography versus cone beam computed tomography. Pediatric dentistry. 2010;32(4):304-9.

10. Cavalcanti M. Diagnóstico por imagem da face. 2 ed: São Paulo: Santos; 2012. $524 \mathrm{p}$.

11. Scarfe WC, Farman AG, Sukovic P. Clinical applications of cone-beam computed tomography in dental practice. J Can Dent Assoc. 2006;72(1):75-80.

12. Primo BT, Andrade MGS, Oliveira HWd, et al. Dentes retidos: novas perspectivas de localização. RFO UPF. 2011;16(1):95-9.

13. Mossaz J, Kloukos D, Pandis N, et al. Morphologic characteristics, location, and associated complications of maxillary and mandibular supernumerary teeth as evaluated using cone beam computed tomography. European journal of orthodontics. 2014;36(6):708-18.
14. Kapila S, Conley RS, Harrell WE. The current status of cone beam computed tomography imaging in orthodontics. Dento maxillo facial radiology. 2011;40(1):24-34

15. Ericson S, Kurol PJ. Resorption of incisors after ectopic eruption of maxillary canines: a CT study. Angle Orthod. 2000;70(6):415-23.

16. Ferreira-Junior O, De Avila LD, Sampieri MB, et al. Impacted lower third molar fused with a supernumerary tooth--diagnosis and treatment planning using cone-beam computed tomography. International journal of oral science. 2009;1(4):224-8.

17. Kato H, Kamio T. Diagnosis and Endodontic Management of Fused Mandibular Second Molar and Paramolar with Concrescent Supernumerary Tooth Using Cone-beam CT and 3-D Printing Technology: A Case Report. The Bulletin of Tokyo Dental College. 2015;56(3):177-84.

18. Scarfe WC, Farman AG. What is cone-beam CT and how does it work? Dental clinics of North America. 2008;52(4):707-30.

19. Reichardt B, Sarwar A, Bartling SH, et al. Musculoskeletal applications of flat-panel volume CT. Skeletal radiology. 2008;37(12):1069-76.

20. Schulze R, Heil U, Gross D, et al. Artefacts in CBCT: a review. Dento maxillo facial radiology. 2011;40(5):265-73.

21. Rodrigues MGS, Alarcón OMV, Carraro E, et al. Tomografia computadorizada por feixe cônico: formação da imagem, indicações e critérios para prescrição. Odontologia Clínico-Científica (Online). 2010;9(2):115-8.

22. Ludlow JB, Davies-Ludlow LE, Brooks SL, et al. Dosimetry of 3 CBCT devices for oral and maxillofacial radiology: CB Mercuray, NewTom 3G and i-CAT. Dento maxillo facial radiology. 2006;35(4):219-26.

23. Mozzo P, Procacci C, Tacconi A, et al. A new volumetric CT machine for dental imaging based on the cone-beam technique: preliminary results. European radiology. 1998;8(9):1558-64.

24. Garib DG, Raymundo Jr R, Raymundo MV, et al. Tomografia computadorizada de feixe cônico (Cone beam): entendendo este novo método de diagnóstico por imagem com promissora aplicabilidade na Ortodontia. Rev Dental Press Ortod Ortop Facial. 2007;12(2):139-56.

25. Farman AG. ALARA still applies. Oral Surgery, Oral Medicine, Oral Pathology, Oral Radiology, and Endodontology. 2005;100(4):395-7.

Recebido em: 19/08/2015 / Aprovado em: 22/09/2015

Nathália de Alencar Valente

Rua Barão de Sertório, 75 - Rio Comprido

Rio de Janeiro/RJ, Brasil - CEP: 20261-050

E-mail: nathaliadeavalente@gmail.com 